\title{
Early Outcomes with a New-generation Humeral Nail: An Institutional Experience
}

\section{Jade Nicolette Chee ${ }^{1 *}$, Bryan Dehao Wang ${ }^{2}$, Andy Kuei Siong Yeo², Thomas Chung Liang Kuo ${ }^{2}$ and Charles Kon Kam King ${ }^{2}$}

${ }^{1}$ Family Medicine, Singhealth Polyclinics, Singapore

${ }^{2}$ Department of Orthopaedic Surgery, Changi General Hospital, Singapore

*Corresponding Author: Jade Nicolette Chee, Family Medicine, Singhealth

Polyclinics, Singapore.

\section{Received: February 17, 2021}

Published: February 27, 2021

(C) All rights are reserved by Jade Nicolette

Chee., et al.

\begin{abstract}
Introduction: The majority of humeral fractures are treated conservatively, while displaced and unstable fractures require surgery. We evaluated our institutional outcome of humeral nailing using a new-generation nailing system. Our surgical technique and operative nuances are described.

We analyzed a cohort of 14 patients with 6 months follow-ups. Radiographic classification (AO/OTA Classification) was performed. Clinical outcome was prospectively charted using American Shoulder and Elbow Surgeons shoulder score (ASES), Oxford and Constant scores. Our secondary outcome was the complication rate of surgery using this new-generation nailing system.

Results: Over one year, 26 patients underwent surgery with complete follow-up data available in 14 patients (nine females). The mean age was 52.4 years old (range, 19-85 years). There were 36\% A-type fractures, 14\% B-type fractures, 21\% C-type fractures, and 29\% shaft fractures. The mean post-operative Constant score was 52.1 (range, 24-100, SD \pm 24.4 ), Oxford score was 33.7 (range, $6-47$, SD \pm 12.7 ) and ASES was 66.1 (range, 11.6 to 98.0, SD \pm 24.9 ). Radiographically, all fractures united by 3-months. The complication rate was $14.3 \%(\mathrm{n}=2 / 14)$.

Conclusion: Our findings show that this new method of nailing was versatile for both simple and complex fractures. In osteoporotic patients, this technique yielded good functional results with minimal complication rates.
\end{abstract}

Keywords: Fracture; Humerus; Intramedullary Nail; Shoulder Trauma; Surgical Technique

\section{Introduction}

Proximal humeral fractures account for around $5 \%$ of all fractures [1]. The frequency of humeral fractures is likely to increase over time as the population ages as a result of both increasing risks of fall and frailty $[2,3]$. The majority of humeral fractures can be managed non-surgically usually with a combination of analgesia, arm sling and immobilization. However, displaced fractures of the humerus would generally require operative management. The main surgical approaches include closed reduction and percutaneous pinning, open reduction and internal fixation with a pre-contoured locking plate, and intramedullary nailing of the humerus.
For more complex fracture patterns, shoulder hemi-arthroplasty and reverse shoulder arthroplasty are indicated.

Plate fixation effectively reduces the fractures and appears to provide excellent biomechanical fixation $[4,5]$. However, this method often leads to devascularisation, scarring and stiffness, especially in the elderly [6]. Primary and secondary screw perforation into the glenohumeral joint are other concerns, with complication rates of up to $35 \%$ with a proximal humerus internal locking plate system [7]. This has led to the introduction of new techniques of fixation, such as intramedullary nailing systems. 
The new-generation humeral nail in this study is indicated for proximal humerus fractures, including 2-4 part surgical neck fractures, proximal humeral fractures with diaphyseal extension, and humeral shaft fractures. This nail is a straight nail with numerous proximal locking options It has a more medial entry point, thus preserving the supraspinatus footprint. In young patients with good bone quality, the outcome is usually satisfactory. However, in the elderly patients with osteoporotic bones, internal fixation frequently leads to failure of fixation, with poor functional results. Lopiz., et al. reported that straight intramedullary nails have comparable union rates to the earlier curvilinear nail designs, with a lower incidence of complications like rotator cuff pain and dysfunction [8].

The aim of this study was to evaluate the clinical and radiographic results of this new humeral nail in our institution.

\section{Materials and Methods}

All consecutive patients who underwent humeral nailing from September 2015 till February 2016 inclusive were included in this analysis. Patients were excluded from the study if they had prior infections, or neuropathic arthropathy. Radiographs were classified using the AO/Orthopaedic Trauma Association (OTA) Classification system for proximal humerus and shaft fractures [9].

Data was collected from patient records on parameters including age, side of surgery, occupation, length of operation, mechanism of injury, past medical history, use of steroids, and time to presentation. Baseline demographics were obtained at the first visit. Outcome results were prospectively collected at follow-up clinic visits.

This new generation humeral nail is a straight, with short and long versions. The nails were made of a titanium alloy (Ti-6Al$7 \mathrm{Nb}$ ), with sizes $8.0 \mathrm{~mm}$ and $9.5 \mathrm{~mm}$ in diameter for the short version, and $7.0 \mathrm{~mm}$ and $8.5 \mathrm{~mm}$ for the long version. The short nail length was $160 \mathrm{~mm}$ in length, and the long nail ranged from 180$315 \mathrm{~mm}$ in length. All surgeries were performed using the multiloc humeral nail (Multiloc humeral nailing system, Synthes, Oberdorf, Switzerland).

Patients were prospectively followed up for a period of 6 months. The study participants were assessed using the American Shoulder and Elbow Surgeons shoulder score (ASES score), the Oxford score, and the Constant score [10-12]. The primary clinical outcome of this study was the post-operative functional outcome score. Complication rates were measured as a secondary outcome of this study.
Data was analysed using IBM SPSS Statistics (Version 20, IBM Corp, Armonk). Categorical variables were presented as percentages. Continuous variables were presented using mean $( \pm$ standard deviation).

Prior to surgical fixation, standard radiographs were performed. These included true antero-posterior, axillary, and scapular Y-views. All patients also had a computer tomographic scan (CT) with three-dimensional reconstruction of their shoulder prior to surgery, in order to assess the personality of the fracture, as well as to adequately exclude head-split fractures of the humeral head [13].

Fractures were classified using the A0/Orthopaedic Trauma Association (OTA) classification (Figure a). For each case, classification was performed with consensus of two surgeons.

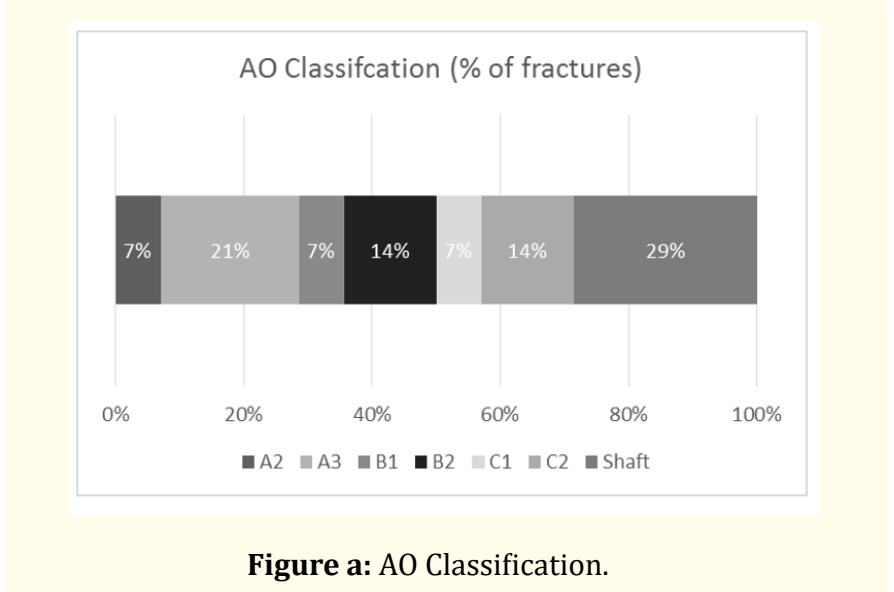

Intra-operatively, patients were placed in a gentle beach chair position (Figure 1), with the C-arm of the image intensifier approaching from the top. To ensure that fluoroscopy was not impaired, patients were positioned at the lateral edge of the table. Fracture alignment, entry position and nail position were assessed with shoulder rotation.

An anterolateral approach was done with an incision starting from the anterolateral edge of the acromion and extending distally. The deltoid muscle was split at the raphe between the anterior and middle third. The subacromial space was cleared of bursal tissue. Number 5 non-absorbable, braided polyethelene terephthalate surgical sutures (Ethibond Excel suture, Ethicon, Somerville, NJ) 


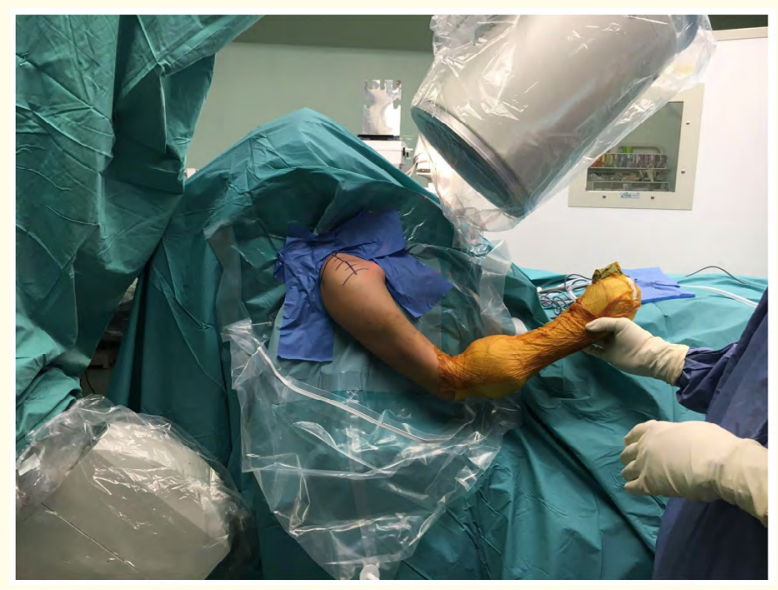

Figure 1: Beach chair position.

were used as traction sutures along the anterior and posterior rotator cuff (Figure 2). In all cases, the axillary nerve was identified by gentle palpation.

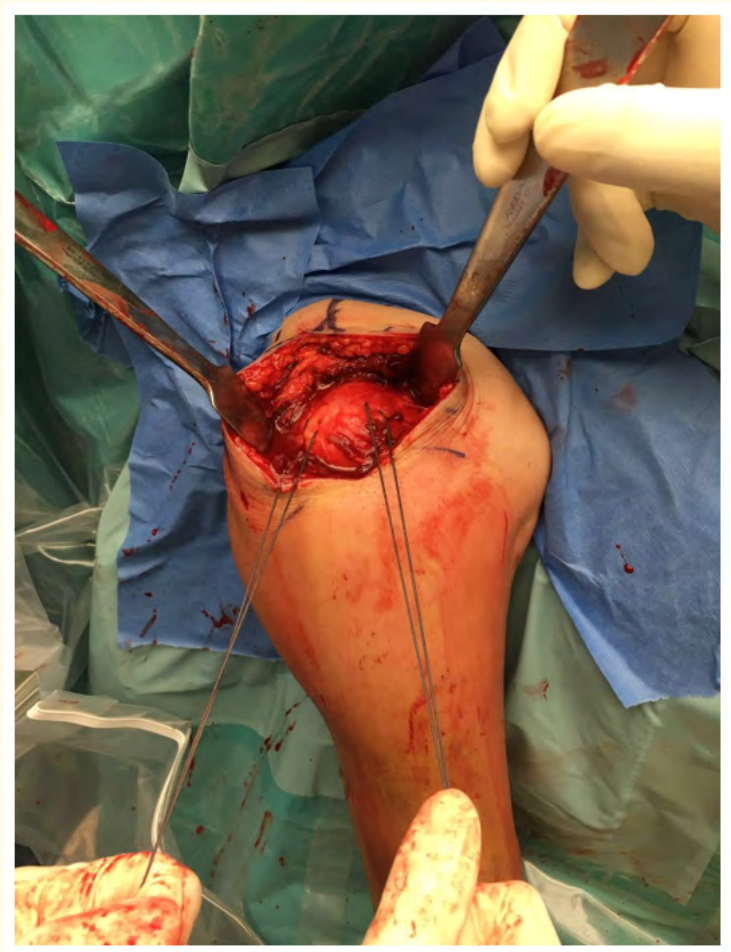

Figure 2: Tagging Ethibond sutures used as a reduction tool.
Fracture reduction was performed using traction sutures to control the individual fragments. In unstable patterns, Kirchner wires were used in addition to hold the reduction as needed. Adequate reduction was confirmed through use of the image intensifier.

The entry point was at the apex of the humeral head in line with the medullary canal in both the anteroposterior (Figure 3a) and lateral (Figure $3 \mathrm{~b}$ ) views. This point was located posterolateral to the biceps tendon and medial to the sulcus between the greater tuberosity and the humeral head. Adequate guide wire placement in both anteroposterior and lateral views were confirmed prior to starting the opening ream.

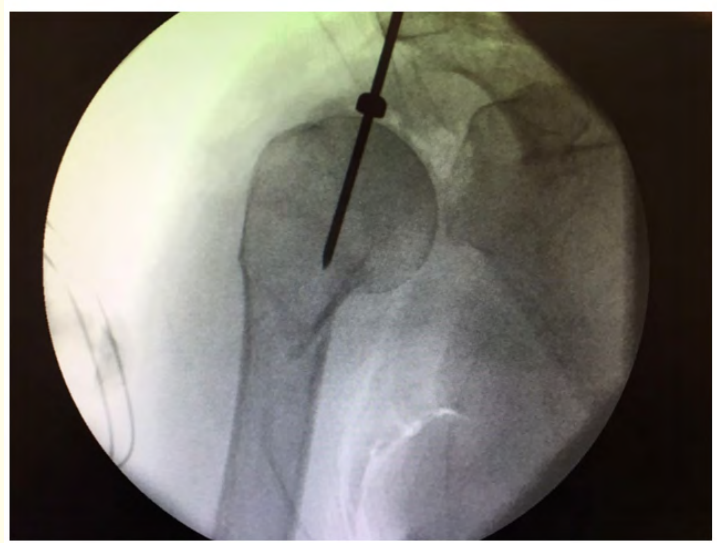

Figure 3a: Entry point (Antero-posterior view).

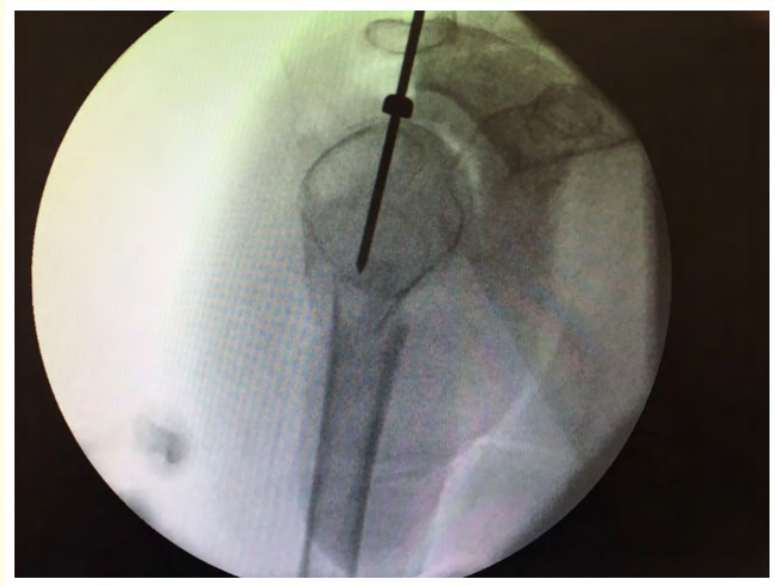

Figure 3b: Entry point (Lateral view). 
After insertion of the guide wire, the nail length and size was measured with a radiographic ruler.

Sequential reaming was performed, and the largest appropriately-sized nail was inserted.

Proximally, a minimum of 3 multiloc screws were used. A screwin screw construct was performed in cases where quality of bone in the humeral head was of concern. Ascending calcar screws were utilized where possible to provide additional posteromedial support. 2 inferomedial screws were inserted distally using a jig. Insertion of the long humeral nail was performed under image intensifier guidance. Prior to insertion, the screw was tied to a synthetic absorbable sterile surgical suture (Vicryl suture, Ethicon, Somerville, NJ) for ease of recovery in event of dislodgement of the screw from the screwdriver during insertion (Figure 4).

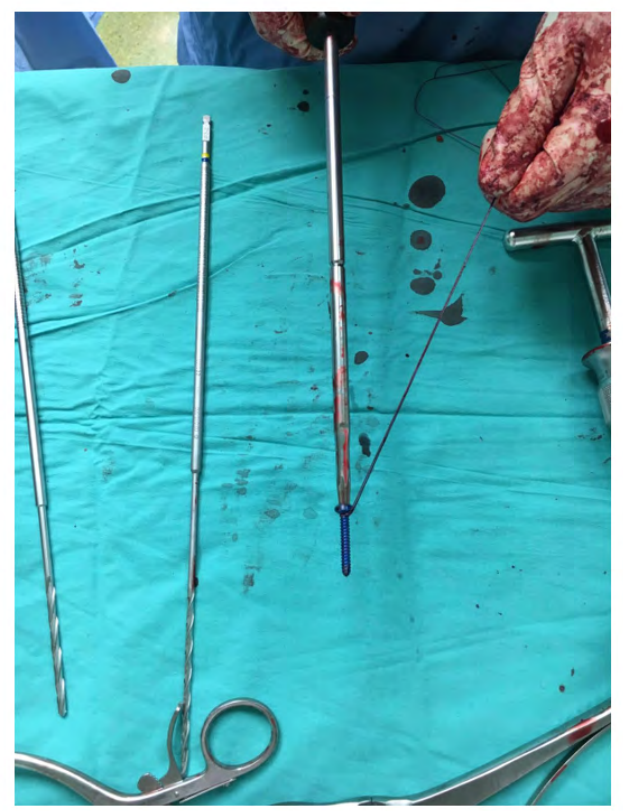

Figure 4: Vicryl stitch tied to distal locking screw.

Care was taken to tag the rotator cuff onto the multiloc screws using the suture holes. The rotator cuff was repaired along with the deltoid split. Anterior-posterior (Figure 5a) and lateral (Figure 5b) post-operative $\mathrm{x}$-rays were taken.

Post-operatively, patients were placed in an arm sling for comfort. For the first 2 weeks, they were allowed pendulum exercises.

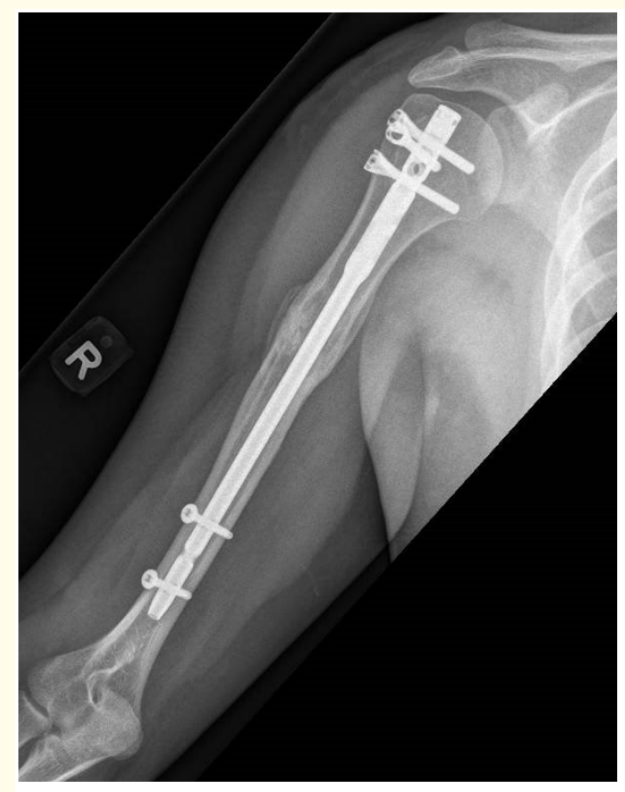

Figure 5a: Post-operative x-ray (anterior-posterior view).

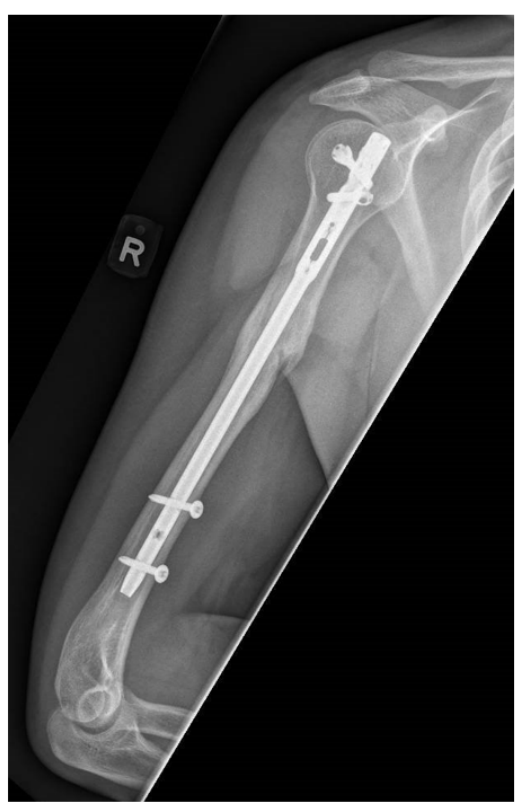

Figure 5b: Post-operative x-ray (lateral view).

Rehabilitation was then increased to passive-assisted exercises for the next 4-weeks, with shoulder abduction of up to 90 degrees. 
Patients were subsequently allowed active mobilization without restrictions after 6 weeks. Weight-bearing and sporting activities were allowed after 3 months. Radiographic evaluations were performed at 1 day, 2 weeks, 6 weeks, 3 months and 6 months postoperatively.

\section{Results}

A total of 26 patients underwent humeral nailing. 12 patients did not fulfill a minimum 6-month post-operative follow-up period, and were excluded from this study. Thus, 14 patients were included in this study. The mean age was 52.4 years old (range, 19-85 years). There were nine females and five males in the study group. All 14 patients were right-hand dominant. 6 patients (42.9\%) were operated on the left humerus, and the remaining 8 patients $(57.1 \%)$ were operated on the right. The average inpatient length of stay was $6.8 \pm 4.7$ days. The length of surgery was $154.3 \pm 40.2$ minutes, with a mean post-operative hospital stay of $3.9 \pm 3.4$ days.

11 patients (78.6\%) had deskbound jobs while 3 patients (21.4\%) had physically active occupations. In our series, all patients had a history of trauma. The mechanism of injury was of high energy in 5 patients (35.7\%). This included road traffic accidents or falls from height. 7 patients (50.0\%) sustained medium energy trauma, and 2 patients (14.3\%) sustained low energy trauma.

Fractures were classified according to the AO classification for proximal humerus and humeral shaft fractures, as summarized in figure a. 36\% had A-type fractures (1 A2, 4 A3 fracture patterns), $14 \%$ had B-type fractures (1 B1, 1 B2 fracture patterns), 21\% had C-type fractures (1 C1, 2 C2 fracture patterns), and 29\% sustained shaft injuries. All fractures had united completely after 3 months.

The mean post-operative Constant score was $52.1 \pm 24.4$ points (range, 24-100). The mean Oxford score was $33.7 \pm 12.7$ points (range, 6-47), and the mean ASES was $66.1 \pm 24.9$ points (range, 11.6 to 98.0 ). Our complication rate was $14 \%(n=2 / 14)$. One patient had varus collapse of the humeral neck, and another one had persistent bicipital pain.

\section{Discussion}

As the frequency of fragility fractures involving the proximal humerus increases [14], we foresee that humeral fixation in the elderly will become increasingly challenging. Our new humeral nail offers screw-in-screw technology for improved fixation in osteoporotic bone [15]. The proximal locking screws target the posteromedial region with stronger bone mineral density, thereby potentially reducing the risk of varus collapse. In our study, $57 \%$ of the patients were aged 57 years and above. This humeral nail has yielded promising results for fixation in osteoporotic bone.

Lanting., et al. found complication rates of $11.9 \%$ for anterograde nailing of humeral fractures, with a $5 \%$ incidence of nonunion and mal-union [16]. In a systematic review of proximal humerus fractures with locking plates, Thanasas., et al. found that the incidence of avascular necrosis was $7.9 \%$, screw cut-out $11.6 \%$ with re-operation rate of $13.7 \%$ [17]. In our study, all fractures united by 3 months ( $\mathrm{n}=14 / 14)$. No intra-articular screw penetration was observed. Our complication rate was $14 \%(n=2 / 14)$. Our functional and radiological results are non-inferior compared with the locking plate system and other nailing systems. Also, we found the sizes and lengths of the humeral nails to be appropriate for our South East Asian population.

The entry point of this nail design being medial to the sulcus provided the additional benefit of preserving the hypovascular region at the insertion of the rotator cuff [18]. Moreover, the straighter design of our nail avoids potential insertion through the fracture site [19]. In our study, no patients complained of postoperative rotator cuff pain $(n=0 / 14)$, indicating that our nail design reduced the incidence of rotator cuff pain in comparison with other intramedullary nailing devices, which reported unsatisfactory outcomes of shoulder function of $25-41 \%$ [20].

This study is not without its limitations. Our study had a small sample size of 14 patients. Also, a 6-month post-operative followup is a relatively short period.

\section{Conclusion}

Improvements in implant design and proximal nailing techniques are ongoing. The basic principles of fracture fixation involve anatomic fracture reduction, stability, preservation of blood supply, and early mobilization. Through this study, we found that this newgeneration humeral nail was easy to insert, with a gentle learning curve. The surgical procedure was also easily reproducible, with a reasonable average inpatient hospital stay of $6.8 \pm 4.7$ days. This new generation humeral nail was versatile, and could be used for both simple and complex fractures. Additionally, this technique yielded good functional results with minimal complication rates even in elderly patients with osteoporotic bones, and is an option to consider with the increase in frequency of fragility fractures involving the proximal humerus in our elderly population. 


\section{Bibliography}

1. Court-Brown CM and Caesar B. "Epidemiology of adult fractures: A review”. Injury 37 (2006): 691-697.

2. Baron JA., et al. "Basic epidemiology of fractures of the upper and lower limb among Americans over 65 years of age". Epidemiology 7 (1996): 612-618.

3. Bengner U., et al. "Changes in the incidence of fracture of the upper end of the humerus during a 30-year period. A study of 2125 fractures". Clinical Orthopaedics 1988231 (1988): 179182.

4. Koval KJ., et al. "Surgical neck fractures of the proximal humerus: a laboratory evaluation of ten fixation techniques". Journal of Trauma 40 (1996): 778-783.

5. Ruch DS., et al. "Fixation of three-part proximal humeral fractures: a biomedical study". Journal of Orthopaedic Trauma 14 (2000): 36-40.

6. Sturzenegger M., et al. "Results of surgical treatment of multifragmented fractures of the humeral head". Archives of Orthopaedic and Traumatic Surgery 100 (1982): 249-259.

7. Brunner F., et al. "Open reduction and internal fixation of proximal humerus fractures using a proximal humeral locked plate: A prospective multicentre analysis". Journal of Orthopaedic Trauma 23.30 (2009): 163-172.

8. Lopiz Y., et al. "Proximal humerus nailing: a randomized clinical trial between curvilinear and straight nails". Journal of Shoulder and Elbow Surgery 23.3 (2014): 369-376.

9. Orthopaedic Trauma Association Committee for Coding and Classification. "Fracture and dislocation compendium". Journal of Orthopaedic Trauma 10 (1996): 1-158.

10. Younis F., et al. "The range of the Oxford Shoulder Score in the asymptomatic population: a marker for post-operative improvement". Annals of The Royal College of Surgeons of England 93.8 (2011): 629-633.

11. Smith M V., et al. "Upper Extremity-Specific Measures of Disability and Outcomes in Orthopaedic Surgery". The Journal of Bone and Joint Surgery. American 94.3 (2012): 277-285.

12. Wylie JD., et al. "Functional outcomes assessment in shoulder surgery”. World Journal of Orthopaedic 5.5 (2014): 623-633.
13. Bahrs C., et al. "Indications for computed tomography (CT-) diagnostics in proximal humeral fractures: A comparative study of plain radiography and computed tomography". BMC Musculoskeletal Disorder 10 (2009): 33.

14. Kannus P., et al. "Osteoporotic fractures of the proximal humerus in elderly Finnish persons: sharp increase in 19701998 and alarming projections for the new millennium". Acta Orthopaedica Scandinavica 71 (2000): 465-470.

15. Rothstock S., et al. "Biomechanical evaluation of two intramedullary nailing techniques with different locking options in a three-part fracture proximal humerus model". Clinical Biomechanics (Bristol, Avon), 27.7 (2012): 686-691.

16. Lanting B., et al. "Proximal humeral fractures: a systematic review of treatment modalities". Journal of Shoulder and Elbow Surgery 17 (2008): 42-54.

17. Thanasas C., et al. "Treatment of proximal humerus fractures with locking plates: a systematic review". Journal of Shoulder and Elbow Surgery 18.6 (2009): 837-844.

18. Park JY and Pandher DS. "Antegrade Humeral Nailing Through the Rotator Cuff Interval: A new entry portal". Journal of Orthopaedic Trauma 22.6 (2008): 419-425.

19. Stedtfeld HW and Mittlmeier T. "Fixation of Proximal Humeral Fractures with an Intramedullary nail: Tips and Tricks". European Journal of Trauma and Emergency Surgery 33.4 (2007): 367-374.

20. Pogliacomi F., et al. "Functional long-term outcome of the shoulder after antegrade intramedullary nailing in humeral diaphyseal fractures". La Chirurgia degli Organi di Movimento 92.1 (2008): 11-16.

\section{Assets from publication with us}

- Prompt Acknowledgement after receiving the article

- Thorough Double blinded peer review

- Rapid Publication

- Issue of Publication Certificate

- High visibility of your Published work

Website: www.actascientific.com/

Submit Article: www.actascientific.com/submission.php Email us: editor@actascientific.com

Contact us: +919182824667 